https://doi.org/10.2478/ jdis-2021-0026

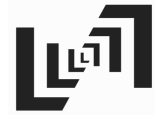

JDIS

Journal of Data and Information Science

\section{New Editorial Board Announced for Journal of Data and Information Science}

We are delighted to announce the new Editorial Board for the Journal of Data and Information Science (JDIS). With some familiar faces staying on and some new ones coming in, the new Editorial Board is appointed for a three-year term, beginning May 10, 2021.

The new board brings together leading experts on the journal's topics from a wide range of institutions and countries/regions. Their expertise would enable $J D I S$ to continue to be a trustworthy academic communication hub. The information about the Editorial Board members is given below and on the journal websites www.jdis.org \& https://sciendo.com/journal/JDIS.

We would like to thank Professor Ying Ding for her effective leadership as one of co-Editors-in-Chief over the past five years. We also express our profound appreciation to other outgoing editorial board members. They have made remarkable contributions to JDIS's development.

The new Editorial Board is as follows:

\section{Co-Editors-in-Chief}

Xiaolin Zhang, National Science Library, Chinese Academy of Sciences, China

Ronald Rousseau, University of Leuven, Belgium

\section{Associate Editor-in-Chief}

Liying Yang, National Science Library, Chinese Academy of Sciences, China

\section{Board Members}

Christine L. Borgman, University of California, USA

Kevin Boyack, SciTech Strategies, Inc., USA

Cong Cao, University of Nottingham Ningbo, China

Dar-Zen Chen, National Taiwan University, Taiwan, China

Cinzia Daraio, Sapienza University of Rome, Italy

Tove Faber Frandsen, University of Southern Denmark, Denmark

Jane Greenberg, Drexel University, USA

Robin Haunschild, Max Planck Institute for Solid State Research, Germany

Xiaojun Hu, Zhejiang University, China

Tao Jia, Southwest University, China

Hamid R. Jamali, Charles Sturt University, Australia

Guangjian Li, Peking University, China

Yuelin Li, Nankai University, China

Wei Liu, Shanghai Library, China

Wei Lu, Wuhan University, China 
Xiaobin Lu, Renmin University, China

Alberto Martin-Martin, University of Granada, Spain

Eustache Megnigbeto, Bureau of Studies and Research in Information Science, Benin

Olga Moskaleva, Saint-Petersburg State University, Russia

Ed Noyons, Centre for Science and Technology Studies, Netherlands

José Miguel Baptista Nunes, Sun Yat-Sen University, China

Han Woo Park, YeungNam University, Korea

Qing Qian, Institute of Medical Information/Medical Library, CAMS, China

Jian Qin, Syracuse University, USA

Elias Sanz-Casado, University Carlos III de Madrid, Spain

Zhesi Shen, National Science Library, Chinese Academy of Sciences, China

Gunnar Sivertsen, Nordic Institute for Studies in Innovation, Research and Education, Norway

Neil Smalheiser, University of Illinois at Chicago, USA

Xinning Su, Nanjing University, China

Shigeo Sugimoto, University of Tsukuba, Japan

Tan Sun, Chinese Academy of Agricultural Sciences, China

Li Tang, Fudan University, China

Mike Thelwall, University of Wolverhampton, UK

Nees Jan van Eck, Centre for Science and Technology Studies, Netherlands

Lili Wang, Maastricht University, Netherlands

Yuefen Wang, Nanjing University of Science and Technology, China

Fang Wang, Nankai University, China

Jevin D. West, Washington University, USA

Dietmar Wolfram, University of Wisconsin-Milwaukee, USA

Dan Wu, Wuhan University, China

Jinshan Wu, Beijing Normal University, China

Yishan Wu, Chinese Academy of Science and Technology for Development, China

Erjia Yan, Drexel University, USA

Guoliang Yang, Institute of Science and Development, Chinese Academy of Sciences, China

Fred Y. Ye, Nanjing University, China

Marcia Lei Zeng, Kent State University, USA

Lin Zhang, Wuhan University, China

Zhixiong Zhang, National Science Library, Chinese Academy of Sciences, China

Dangzhi Zhao, University of Alberta, Canada

Yuxiang Zhao, Renmin University, China 